\title{
Performance of new gellan gum hydrogels combined with human articular chondrocytes for cartilage regeneration when subcutaneously implanted in nude mice
}

\author{
J. T. Oliveira ${ }^{1,2 *}$, T. C. Santos ${ }^{1,2}$, L. Martins ${ }^{3}$, M. A. Silva ${ }^{1,2}$, A. P. Marques $^{1,2}$, A. G. Castro ${ }^{3}$, \\ N. M. Neves ${ }^{1,2}$ and R. L. Reis ${ }^{1,2}$ \\ ${ }^{1} 3$ Bs Research Group - Biomaterials, Biodegradables and Biomimetics, University of Minho, Headquarters of the European Institute of \\ Excellence on Tissue Engineering and Regenerative Medicine, AvePark, 4806-909 Taipas, Guimarães, Portugal \\ ${ }^{2}$ Institute for Biotechnology and Bioengineering (IBB), PT Associated Laboratory, Guimarães, Portugal \\ ${ }^{3}$ Life and Health Sciences Research Institute (ICVS), School of Health Sciences, University of Minho, 4710-057 Braga, Portugal
}

\begin{abstract}
Gellan gum is a polysaccharide that has been recently proposed by our group for cartilage tissueengineering applications. It is commonly used in the food and pharmaceutical industry and has the ability to form stable gels without the use of harsh reagents. Gellan gum can function as a minimally invasive injectable system, gelling inside the body in situ under physiological conditions and efficiently adapting to the defect site. In this work, gellan gum hydrogels were combined with human articular chondrocytes (hACs) and were subcutaneously implanted in nude mice for 4 weeks. The implants were collected for histological (haematoxylin and eosin and Alcian blue staining), biochemical [dimethylmethylene blue (GAG) assay], molecular (real-time PCR analyses for collagen types I, II and X, aggrecan) and immunological analyses (immunolocalization of collagen types I and II). The results showed a homogeneous cell distribution and the typical round-shaped morphology of the chondrocytes within the matrix upon implantation. Proteoglycans synthesis was detected by Alcian blue staining and a statistically significant increase of proteoglycans content was measured with the GAG assay quantified from 1 to 4 weeks of implantation. Real-time PCR analyses showed a statistically significant upregulation of collagen type II and aggrecan levels in the same periods. The immunological assays suggest deposition of collagen type II along with some collagen type I. The overall data shows that gellan gum hydrogels adequately support the growth and ECM deposition of human articular chondrocytes when implanted subcutaneously in nude mice. Copyright $\odot 2009$ John Wiley \& Sons, Ltd.
\end{abstract}

Received 26 January 2009; Revised 12 May 2009; Accepted 15 May 2009

Keywords cartilage; tissue engineering; hydrogel; adipose stem cells; in vivo; natural biomaterial

\section{Introduction}

Cartilage is a supporting connective tissue, made up broadly of proteins, polysaccharides and chondrocytes,

\footnotetext{
*Correspondence to: J. T. Oliveira, 3Bs Research GroupBiomaterials, Biodegradables and Biomimetics, Department of Polymer Engineering, University of Minho, Headquarters of the European Institute of Excellence on Tissue Engineering and Regenerative Medicine, AvePark, Zona Industrial da Gandra, S. Cláudio do Barco, 4806-909 Caldas das Taipas, Guimarães, Portugal.E-mail: joao.oliveira@dep.uminho.pt
}

that develops an important role in maintaining mobility and a smooth gliding surface of joints in the skeleton. This function is mainly assured by the extracellular matrix that surrounds the chondrocytes, which is able to withstand physical deformation and facilitate tissue function. Chondrocytes synthesize and maintain this extracellular matrix, producing especially collagen type II and aggrecan, which confer articular cartilage its tensile and compressive resistance, respectively. Cartilage has a low metabolism that may constitute a serious barrier to normal locomotion when the tissue is traumatized or 
degenerated. As healthy cartilage is only synthesized to a short extent, and frequently this neocartilage presents a much more pronounced fibrocartilaginous nature than hyaline cartilage, pathological scenarios are triggered and life quality is highly diminished (Temenoff and Mikos, 2000; Ge et al., 2006; Mano and Reis, 2007). Various ways to address these problems have been suggested, such as debridement or drilling, but the outcome is still not satisfactory (Hunt et al., 2002; Jackson and Dieterichs, 2003). Mosaicplasty (Hangody and Fules, 2003; Jakob et al., 2002), although being a widespread surgical technique for treating a cartilage lesion, creates a problem of reconstruction of the cartilage collection site while repairing the original defect. Among the different alternatives proposed was tissue engineering of cartilage tissues (Freed et al., 1993; Vacanti and Vacanti, 1994; Sittinger et al., 1994; Oliviera et al., 2006, 2008a), using biomaterials, cells and/or bioactive agents. One of the first definitions of tissue engineering was proposed by Langer and Vacanti (1993). Since then, tissue-engineering concepts have evolved and progressed through several lines of study that range from nanotechnology-inspired systems to rapid prototyping methods aimed at producing hybrid structures (Capito et al., 2008; Moroni et al., 2006; Hendriks et al., 2007; Tuzlakoglu et al., 2005; Andersson and van den Berg, 2004). Also, the types of biodegradable materials chosen passed from those already employed in clinical procedures (in applications such as sutures, stents, etc.) to others used in environmental applications (Videcki et al., 2005) and the food industry (Mao et al., 2000). Biomaterials used in regenerative medicine are frequently divided in terms of their natural or synthetic origins. Examples of the first include alginate (Rowley et al., 1999), hyaluronic acid (Cortivo et al., 1991), chitosan (Malafaya et al., 2008) and starch (Gomes et al., 2008), and of the second, polyglycolic acid (Mooney et al., 1996), polylactic acid (Cao et al., 1997) and polyethylene oxide (Hubbell et al., 1991). Gellan gum is a biomaterial of natural origin recently proposed for applications in the cartilage regeneration field (Oliviera et al., 2008b, 2008 c). It is a polysaccharide produced by bacterial fermentation and its basic structural unit is composed of glucose, rhamnose and glucuronic acid residues. Gellan gum is able to form gels with differences in mechanical properties, from soft and elastic to hard and brittle, through an ionotropic gelation mechanism (Kang et al., 1982; Grasdalen and Smidsrod, 1987). Other interesting characteristics of the material include its heat and acid resistance, gel formation under mild conditions without using harsh reagents and its non-cytotoxic behaviour (Oliviera et al., 2008d). Our group has suggested (Oliviera et al., 2008c; 2008d) the use of gellan gum as an encapsulating and support agent of different cells towards the formation of a functional cartilaginous tissue. Human articular cartilage chondrocytes were encapsulated and cultured in vitro in optimized injectable gellan gum systems for total periods of 8 weeks, maintaining their viability and synthesizing hyaline-like extracellular matrix components, mainly collagen type II and aggrecan.
In this work, gellan gum hydrogels were combined with human articular chondrocytes acting as encapsulating agents and supports for their development. Gellan gum discs were formed and subcutaneously implanted in the back of nude mice for 4 weeks periods. The in vivo results showing the formation of a cartilage tissue of hyaline nature suggest that these systems may be potentially used in the repair of cartilage lesions.

\section{Materials and methods}

\subsection{Human articular chondrocytes isolation and expansion}

Articular cartilage was harvested from the femoral head and condyles macroscopically healthy parts of adult patients (aged 40-65 years) undergoing replacement surgery based on a protocol previously described by Crawford and Dickenson (2004). This was performed within the scope of a protocol established with the Hospital de S. Marcos, Braga, Portugal, approved by its Ethical Committee and always dependent upon the patients' informed consent. Human chondrocytes were isolated by enzymatic digestion with posterior collection. The human articular cartilage, freed from all surrounding tissue, was placed in a Petri dish containing sterile phosphate-buffered saline (PBS) and cut into square slices of $5 \mathrm{~mm}^{2}$ and thickness $2-3 \mathrm{~mm}$. The pieces were washed in sterile PBS solution, immersed in $20 \mathrm{ml}$ trypsin-EDTA solution and incubated for $30 \mathrm{~min}$ at $37^{\circ} \mathrm{C}$ on a rotator. Trypsin was removed and the pieces washed with basic DMEM (Sigma-Aldrich Co., USA). Then, $20 \mathrm{ml}$ filter-sterilized collagenase type II (SigmaAldrich) solution $(2 \mathrm{mg} / \mathrm{ml})$ in basic medium was added, and the mixture incubated for approximately $10 \mathrm{~h}$ at $37^{\circ} \mathrm{C}$ on a rotator. The digested tissue and cell suspension solution was centrifuged at $1200 \mathrm{rpm}$ for $8 \mathrm{~min}$ and the supernatant removed. The cell pellet was washed and centrifuged twice with PBS and the cells counted using a haemocytometer. Chondrocytes were then collected by centrifugation and resuspended in expansion medium consisting of Dulbecco's modified Eagle's medium (SigmaAldrich) containing $10 \mathrm{mM}$ HEPES buffer, $\mathrm{pH}$ 7.4, $1 \%$ antibiotic (antibiotic-antimycotic; Gibco 15240), $20 \mathrm{~mm}$ L-alanyl glutamine, $1 \times$ MEM non-essential amino acids and $10 \% \mathrm{v} / \mathrm{v}$ fetal bovine serum (FBS, heatinactivated; Biochrom, Berlin, Germany), supplemented with $10 \mathrm{ng} / \mathrm{ml}$ basic fibroblast growth factor (bFGF; PeproTech, UK). Human articular chondrocytes were plated into tissue culture flasks and incubated at $37^{\circ} \mathrm{C}$ in a humidified atmosphere of $5 \% \mathrm{CO}_{2}$ in air for expansion.

\subsection{Human articular chondrocytes encapsulation in gellan gum hydrogels}

Human articular chondrocytes were expanded and encapsulated at passage 2 in gellan gum hydrogels using 
the following procedure. Briefly, gellan gum powder (G1910; Sigma-Aldrich) was mixed with sterile distilled water under constant stirring at room temperature to obtain a final concentration of $1.25 \% \mathrm{w} / \mathrm{v}$. The solution was progressively heated to $90^{\circ} \mathrm{C}$ and kept at this temperature for $20-30 \mathrm{~min}$. The temperature was progressively decreased to $42^{\circ} \mathrm{C}$ and stabilized under constant stirring. Human articular chondrocytes were detached by trypsinization, mixed with culture medium and centrifuged at $1200 \mathrm{rpm}$ for $8 \mathrm{~min}$. The supernatant was removed and the cells were resuspended in sterile PBS solution, counted using a haemocytometer and finally centrifuged as before. The cell number was calculated so that the final concentration after encapsulation was $5 \times 10^{6} \mathrm{cells} / \mathrm{ml}$. The supernatant was discarded and the cells pellet kept at the bottom of the tube were resuspended in PBS. The gellan gum $1.25 \% \mathrm{w} / \mathrm{v}$ solution was extensively mixed with the chondrocytes suspension for complete homogeneous dispersion within the gel. Gellan gum with the encapsulated cells was allowed to gel in a cylindrical mould for 2-3 min. Discs of $3 \mathrm{~mm}$ diameter $\times 3 \mathrm{~mm}$ height were cut using a sterile blade and kept in sterile PBS before the implantation procedure. Gellan gum discs with no cells encapsulated were also prepared, using the same procedure, and used as controls.

\subsection{In vivo subcutaneous implantation in nude mice}

Six 4 week-old female Balb/C nude mice (Charles River Laboratories Inc., USA), female with an average weight of $20 \mathrm{~g}$, were anaesthetized with a mixture of ketamine $\left(1.2 \mathrm{mg}\right.$ /mouse s.c.; Imalgene ${ }^{\circledR} 1000$, Merial, Lyon, France) and medetomidine $\left(20 \mu \mathrm{g} / \mathrm{mouse}\right.$ s.c.; Domitor ${ }^{\circledR}$, Orion Corporation, Finland) prepared in physiological serum. After the confirmation of analgesia/anaesthesia, two incisions were made (reaching a maximum of $1.5 \mathrm{~cm}$ each), one in the intrascapular region and another in the lumbar region. With the help of forceps, two side pockets were created through each of the incisions and gellan gum discs with encapsulated chondrocytes and with no encapsulated cells (control) were subcutaneously implanted. Four discs were implanted per animal, two on the anterior region and other two on the posterior region. The incision sites were sutured and the mice transferred to heating recovery compartments and, when recovery from analgesia/anaesthesia was confirmed, they were returned to their respective compartments and kept under food and drink ad libitum. After 1 week and 4 weeks post implantation, the mice were euthanized $(n=3$ for each time point) by exposure to a saturated carbon dioxide environment and the gellan gum discs were surgically recovered and processed for histological, biochemical and molecular analyses. The animal tests were performed after analysis of the experimental plan and approval by the Local Ethical Committee.

\subsection{Histology}

Common histological analysis was performed on $4 \mu \mathrm{m}$ thickness sections of the explants collected at different periods of culture. Haematoxylin and eosin (H\&E) staining was conducted to observe general cell morphology and overall distribution, and Alcian blue was performed to evaluate extracellular matrix components deposition, viz. proteoglycans (glycosaminoglycans). Briefly, the constructs were carefully dissected from the subcutaneous tissue of nude mice and collected in Eppendorf tubes. They were immediately fixed in formalin for 30-40 $\mathrm{min}$ and washed in PBS. Histological processing was conducted by dehydrating the samples in increasing ethanol concentrations, embedding them in paraffin and cutting sections for posterior analysis using a Leica RM2155 microtome (Leica Microsystems, Nusslock GmbH, Germany). H\&E staining was performed using an automatic processor according to in-house methodology (Leica TP1020-1, Leica MicroSystems) and Alcian blue staining was performed using standard histological methods. The slides were washed afterwards in distilled water, dehydrated through increasing ethanol concentrations and finally cleared in xylene substitute and mounted using Microscopy Entellan ${ }^{\circledR}$ (Merck \& Co. Inc., USA) for observation.

\subsection{Quantification of proteoglycan content}

Proteoglycans were determined by measuring the level of sulphated glycosaminoglycans (GAGs), using 1,9dimethylmethylene blue (DMB) metachromatic assay, as previously described by Kafienah and Sims (2004). GAG levels can be quantified in solution using DMB, since the mechanical entanglement of this reagent with GAGs generates a peak shift at $A_{525-530}$ that can be measured spectrophotometrically. Briefly, the constructs were carefully dissected from the subcutaneous tissue of nude mice and collected in Eppendorf tubes. The samples were ground with a mortar and pestle and immersed in a digestion solution with papain and $\mathrm{N}$-acetyl cysteine at $60^{\circ} \mathrm{C}$ for approximately $3 \mathrm{~h}$. The tubes were centrifuged at $13000 \mathrm{rpm}$ for $10 \mathrm{~min}$ and the supernatant was collected for biochemical analysis. A chondroitin sulphate standard solution was prepared in water and kept at $4{ }^{\circ} \mathrm{C}$. The samples and the chondroitin sulphate standards were placed in a 96-well round-bottomed plate, DMB solution was added to each well, and the optical density was measured using a microplate reader at $530 \mathrm{~nm}$. Statistical analyses were conducted using a two-sample $t$-test, assuming unequal variances for $n=3$.

\subsection{Real-time PCR (collagen types I, II and X, aggrecan)}

The constructs were carefully collected upon dissection of the subcutaneous tissue of nude mice, immersed 
in TRIzol $^{\circledR}$ (Invitrogen, USA) and quickly and stored at $-80^{\circ} \mathrm{C}$ until the analysis was performed. RNA was extracted using TRIzol and more details can be found in the technical datasheet provided by the supplier. Briefly, triplicates of each condition were ground and mechanically homogenized with a mortar and pestle in TRIzol. Chloroform was then added and the samples centrifuged to establish a three-phase composition in the tube, then the aqueous phase was collected to a new tube and mixed with isopropanol. The samples were again centrifuged, the supernatant discarded and the pellet washed with $75 \%$ ethanol. The samples were again centrifuged, allowed to dry in air and suspended in ultrapure water for posterior analysis. The amounts of isolated RNA and A260:280 ratio were determined using Nanodrop ND-1000 Spectrophotometer (NanoDrop Technologies Inc., USA). After these determinations, RNA from each sample was reverse-transcribed into cDNA, using the IScript ${ }^{\mathrm{TM}}$ cDNA synthesis kit (Bio-Rad Laboratories, USA) in a BioRad CFX96 real-time PCR detection system. Cartilage-related markers were chosen to evaluate the chondrogenic phenotype of the cultured systems. These included collagen types I, II and $\mathrm{X}$ and aggrecan, using GAPDH as the housekeeping gene for normalization. The expression of each gene was normalized to the GAPDH value in that sample. The relative gene expression quantification was performed using the $2^{-\Delta \Delta \mathrm{Ct}}$ (Livak) method, considering that:

$$
2^{-\Delta \Delta \mathrm{Ct}}=\text { Normalized expression ratio }
$$

All the primer sequences were generated using Primer3 software (http://frodo.wi.mit.edu/) and acquired from MWG Biotech, Germany. More details can be found in Table 1. Real-time PCR was performed using a BioRad CFX96 real-time PCR detection system and SYBR Green $\mathrm{IQ}^{\mathrm{TM}}$ Supermix (Bio-Rad) to detect amplification variations. The analyses of the results were performed with CFX Manager Software, version 1.0 (Bio-Rad). Statistical analyses were conducted using a two-sample $t$-test, assuming unequal variances for $n=3$.

\subsection{Immunolocalization of collagen types I and II}

Collagen types I and II were detected immunohistochemically, using monoclonal antibodies against collagen types I and II (Southern Biotechnology, UK), using the Vectastain ${ }^{\circledR}$ Universal Elite ABC Kit PK-7200 (Vector
Laboratories Ltd, UK) and DAB Substrate Kit for Peroxidase SK-4100 (Vector Laboratories) according to the suppliers' instructions. Briefly, paraffin sections of the collected explants were deparaffinized and hydrated through decreasing ethanol concentrations. The sections were treated with $3 \%$ hydrogen peroxide in $50 \%$ methanol for $5 \mathrm{~min}$, washed in PBS buffer and incubated in prediluted blocking serum. The incubation with the primary antibodies and negative control followed (collagen type I, $1: 100$; collagen type II, $1: 20$; normal horse serum, $1: 100)$. The remaining protocol is as described in the Vectastain ${ }^{\circledR}$ Elite $A B C$ Kit. The sections were further incubated with the DAB substrate at room temperature until suitable staining develops, according to the supplier's instructions. The sections were counterstained with neutral red, dehydrated through increasing ethanol concentrations and finally cleared in Xylene Substitute ${ }^{\circledR}$ (National Diagnostics, USA) and mounted using Microscopy Entellan ${ }^{\circledR}$ (Merck, USA) for observation.

\section{Results}

\subsection{Histology}

The explants collected after 1 and 4 weeks of implantation were analysed using histological methods. H\&E staining was performed on the sections of the explants, since it can provide relevant information on cell morphology and distribution within the gel matrix. It was observed that the cells had been homogeneously distributed throughout the whole hydrogel, while also exhibiting the typical roundshaped morphology of native articular chondrocytes. An increase in cell mass was noticed after 4 weeks of culture (Figure 1A), with individual chondrocytes giving rise to clusters of two or three cells. Sulphated glycosaminoglycans are important components of the native articular cartilage extracellular matrix, due to their water-retaining ability that highly contributes to the mechanical functionality of the tissue. Sulphated glycosaminoglycans were detected in histological sections of the explants of the implanted gellan gum systems, using Alcian blue staining, mostly after 4 weeks of implantation (Figure 1B). The staining evolved from a more orthochromatic nature in the early periods to a pronounced metachromatic staining after 4 weeks of implantation (Figure 1B). The positive staining was localized in the pericellular regions of chondrocyte clusters, its presence and intensity being quite regular throughout the gellan gum matrix. It should also be

Table 1. Primers used for real-time PCR evaluation of human articular chondrocytes gene expression

\begin{tabular}{llll}
\hline Gene & Accession No. & \multicolumn{1}{c}{ Left primer } & Right primer \\
\hline Collagen type I & NM_000089 & CTGCAAGAACAGCATTGCAT & GGCGTGATGGCTTATTTGTT \\
Collagen type II & NM_001844 & TCACGTACACTGCCTGAAG & TGCAACGGATTGTGTTGTTT \\
Aggrecan & NM_001135 & ACAGCTGGGGACATTAGTGG & GTGGAATGCAGAGGTGGTTT \\
Collagen type X & NM_000493 & AATCCCACAGGCATAAAAG & AGGACTTCCGTAGCCTGGTT \\
GAPDH & NM_002046 & GAGTCAACGGATTGGTCGT & TTGATTTGGAGGGATCTCG \\
\hline
\end{tabular}




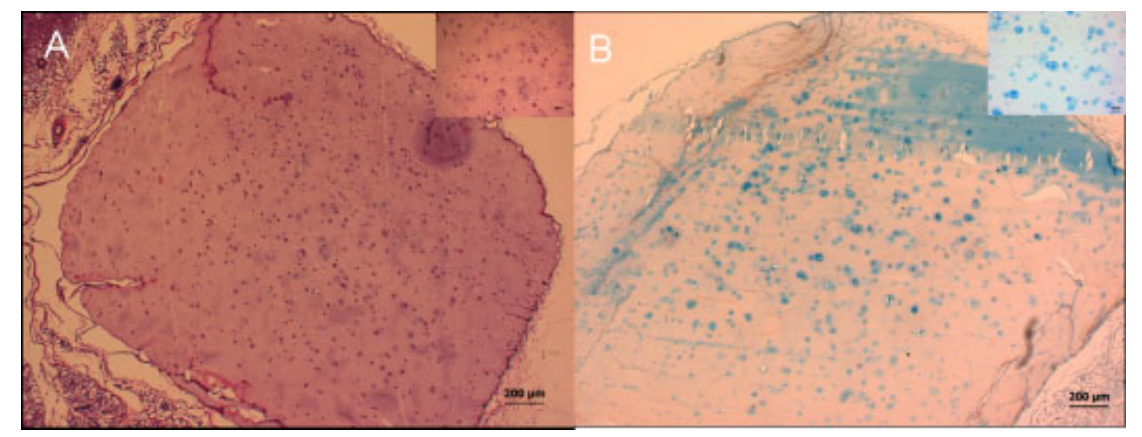

Figure 1. H\&E (A) and Alcian blue (B) staining of histological sections of the explanted gellan gum hydrogels with human articular chondrocytes after 4 weeks of culture. The cells divided in clusters of two or three cells and presented the typical round-shaped morphology of native articular chondrocytes. Metachromatic staining, mostly in the pericellular regions, can be observed indicating the deposition of extracellular matrix proteoglycans (glycosaminoglycans) (B)

mentioned that the discs maintained their structural integrity upon microscopical observation and were well integrated with the surrounding tissues.

\subsection{Quantification of proteoglycan content}

Following the qualitative analysis performed with Alcian blue staining on histological sections of the gellan gum hydrogels-human articular chondrocytes tissue explants, the proteoglycan content of the constructs was quantitatively evaluated using the GAG assay. The glycosaminoglycan content was found to increase steadily from 1 to 4 weeks of implantation, this variation being statistically significant (Figure 2). An increase of approximately 2.4-fold was measured in gellan gum tissue-engineered constructs collected after 4 weeks compared with 1 week of implantation. These results are in accordance with, and reinforce the positive identification of, sulphated glycosaminoglycans found in the histological analysis.

\subsection{Real-time PCR (collagen types I, II and X, aggrecan)}

Real-time PCR was used to quantitatively assess the upand downregulation of genes typically associated with chondrocytes and cartilage tissue formation (Figure 3). Collagen types I, II, $\mathrm{X}$ and aggrecan are common

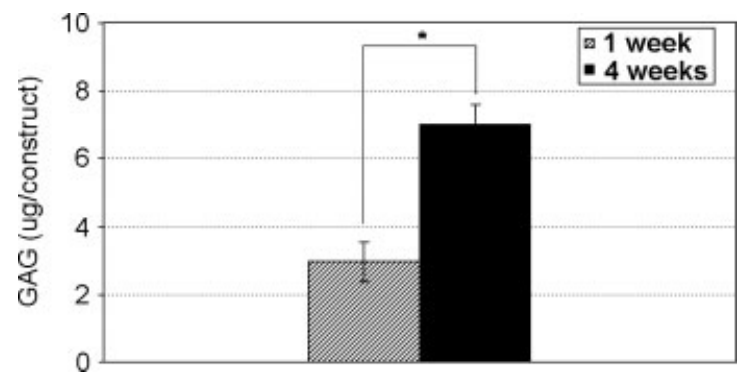

Figure 2. Graphical representation of the results obtained from DMB assay for glycosaminoglycans (GAGs) quantification of the explants after 1 and 4 weeks of implantation. A statistically significant increase was observed from 1 to 4 weeks $(p<0.05)$
ECM constituents present at different stages of the chondrogenic process, and the transcription of their genes was evaluated after 1 and 4 weeks of implantation. Among those, collagen type II and aggrecan are considered to be the two major and most important constituents of hyaline cartilage ECM, since they are responsible for the mechanical functionality of the tissue. Collagen type I is associated with the dedifferentiation period that frequently occurs in $2 \mathrm{D}$ culturing and is a reflection of the poor hyaline nature of the tissue formed (Benya and Shaffer, 1982; Cancedda et al., 1995). Collagen type X is a marker associated with hypertrophic chondrocytes and matrix mineralization, and its presence is a poor indicator of the formation of a stable hyaline-like ECM (von der Mark et al., 1992). The graphical representation of the molecular analyses data shows a statistically significant increase of both collagen type II and aggrecan of approximately 230-fold and six-fold, respectively, from 1 to 4 weeks of implantation. Collagen type I and collagen type $\mathrm{X}$ were not detected until the end of the experiments.

\subsection{Immunolocalization of collagen types I and II}

As previously mentioned, collagen type II is the most important major protein produced by chondrocytes

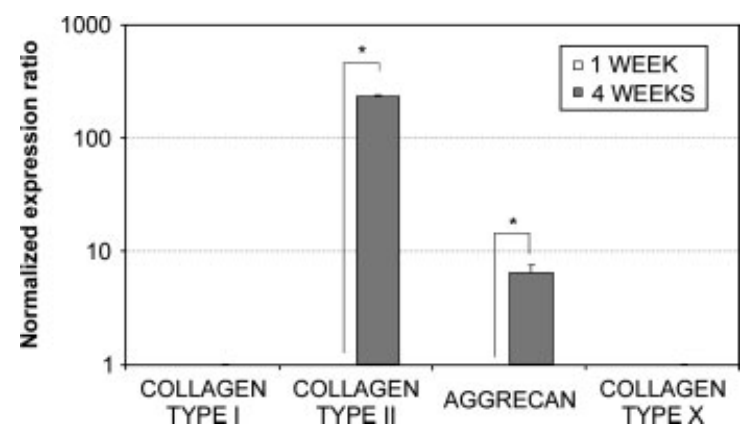

Figure 3. Real-time PCR analyses results for collagen type I, collagen type II, aggrecan, and collagen type $\mathrm{X}$ during the 4 weeks of implantation. Collagen type II and aggrecan presented statistically significant increases from 1 to 4 weeks, indicating the hyaline-like nature of the newly formed tissue $(p<0.05)$ 


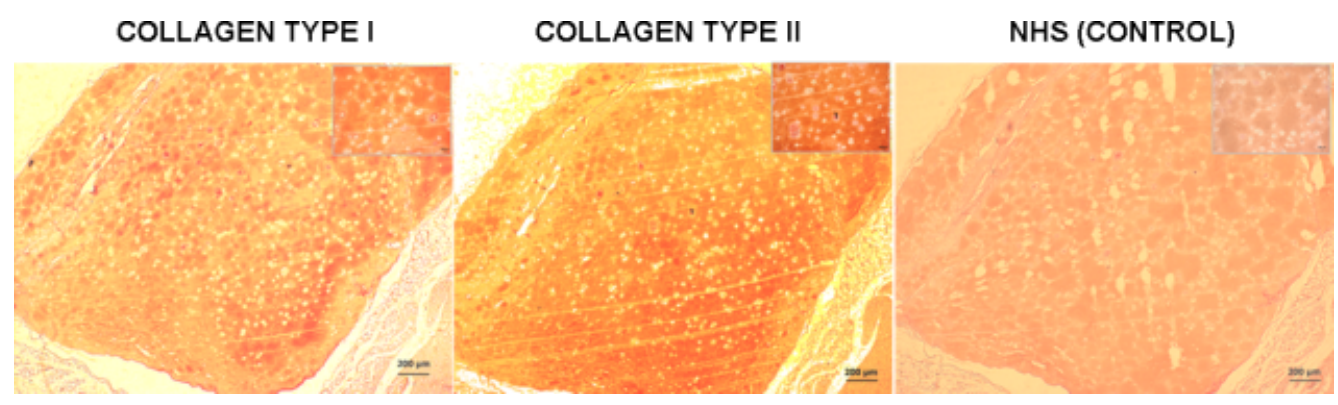

Figure 4. Immunolocalization of collagen types I and II in histology sections of the explanted gellan gum hydrogels-human articular chondrocytes systems after 4 weeks. Images show collagen type I, collagen type II and normal horse serum (negative control) (left to right, respectively)

in articular cartilage, with key functions in the weight-bearing ability and shock-absorbing properties of the tissue. The analysis of the immunostaining profiles (Figure 4) indicates that both collagen type I and type II are present in the explants when compared with the negative control (normal horse serum). No striking difference is encountered between collagen types I and II, although some stronger staining may be noticed for type II. However, the qualitative evaluation is not simple, mostly due to the background staining of the hydrogel that is also present in the negative control. The identification of collagen types I and II, with a suggested prevalence of the latter, is another evidence of the hyaline-nature ECM of the tissue-engineered constructs and is in agreement with previous molecular data obtained from real-time PCR analyses that showed an upregulation of collagen type II mRNA.

\section{Discussion}

Gellan gum hydrogels have been previously suggested by our group (Oliviera et al., 2008b, 2008c, 2008d) for applications in cartilage regeneration, since it possesses adequate material properties for these purposes. It can be used efficiently as a minimally invasive injectable system, able to deliver and encapsulate cells and to support their development in vitro. In those studies, human articular chondrocytes were encapsulated in injectable gellan gum hydrogels and cultured in vitro for extensive periods. The chondrocytes were viable and formed a hyaline-like ECM composed of collagen type II and aggrecan. The evaluation of the in vivo response upon subcutaneous implantation of these biomaterials was conducted in mice, revealing good integration with the surrounding tissues and the presence of a residual fibrotic capsule. The preliminary data collected with these hydrogels was quite promising regarding their potential use in the treatment of cartilage pathologies, and further in vivo studies were set up to validate this hypothesis, which are presented in this paper (Oliveira et al., 2008d). ${ }^{32}$

In this study, gellan gum hydrogels were used to encapsulate human articular chondrocytes and were processed in the form of discs to be subcutaneously implanted in the back of nude mice. The main objective was to assess the formation of a hyaline-like cartilage tissue and to conclude on the adequacy of these systems to generate a functional tissue-engineered construct with in vivo relevance in the future, using histological, biochemical and molecular characterization techniques. H\&E staining of histological sections revealed a homogeneous distribution of the chondrocytes within the gellan gum matrix, with the cells exhibiting the common round-shaped phenotype characteristic of native chondrocytes. During the implantation time, the chondrocytes exhibited active division (Figure 1B), which is a good indication of their viability and growth supported by the gellan gum hydrogels. Although these are important aspects to take into account when following cartilage tissue-engineering approaches, the essential feature is the formation of a stable and functional tissue, more precisely an ECM, that should be similar to the native one. When referring to the mimetization of the native articular cartilage ECM, efforts are frequently directed to the creation of a matrix composed mostly of collagen type II and aggrecan. In fact, these two molecules are responsible for the important functions that cartilage has in normal mobility and joint movement. Articular cartilage has a physical role in the tissue load-bearing properties, mainly due to the interactions of water, ions and aggrecan molecules within the collagenous meshwork. The collagen type II confers tensile strength and the aggrecan molecules provide compressive stiffness to the tissue (Ge et al., 2006; Knudson and Knudson, 2001). The study of the deposition and quantification of proteoglycans, where aggrecan is the most abundant, was performed with Alcian blue staining and the GAG assay. The staining profile improved from 1 to 4 weeks of implantation, mostly located in the pericellular regions (Figure 1). The metachromatic staining was more pronounced at the last time point, indicating a higher deposition of proteoglycans by this stage, thereby implying that the production of these molecules increased during the course of the experiments. These results are in accordance with the quantitative analysis, which showed a statistically significant increase from 1 to 4 weeks of implantation and reinforced the fact that proteoglycans were being synthesized by the encapsulated human articular chondrocytes. Real-time PCR analyses were performed to quantify collagen types I, II and X and aggrecan mRNA levels throughout the time course of the experiments. A statistically significant 
upregulation of both collagen type II and aggrecan mRNA levels was observed from 1 to 4 weeks of implantation. As previously mentioned, these are the most important components of the articular cartilage ECM and the transcription of both genes emphasizes that hyaline cartilage ECM is being deposited, opening interesting prospects regarding future applications of this biomaterial. To this can be added that collagen type $\mathrm{I}$ and collagen type $\mathrm{X}$ are downregulated from 1 to 4 weeks. When in a two-dimensional (2D) environment chondrocytes dedifferentiate, losing their round-shaped phenotype and decreasing the production of collagen type II and aggrecan, while increasing the production of type I collagen (Cancedda et al., 1995; Vunjak-Novakovic and Freed, 1998). The presence of collagen type I is therefore a negative indicator for hyaline-like cartilage ECM formation. Collagen type $\mathrm{X}$ is frequently expressed by hypertrophic chondrocytes and is associated with matrix mineralization. For instance, Kirsch et al. (1992) observed that collagen type $\mathrm{X}$ synthesis is normally linked to an increase of intracellular calcium and deposition of calcium mineral, ultimately leading to matrix calcification. Chondrocytes expressing this marker are usually part of the deep zone of articular cartilage and its presence indicates an hyperthrophic state of the chondrocytes, which is not favourable when attempting to obtain an articular cartilage-like ECM (Girkontaite et al., 1996). The absence of both collagen type I and collagen type $\mathrm{X}$ suggests that chondrocytes were able to redifferentiate after the previous $2 \mathrm{D}$ expansion period in vitro but were kept in that stage, since no collagen type $\mathrm{X}$ typical of hyperthrophic chondrocytes could be detected. Finally, to complement the analyses, immunological localization of collagen type I and collagen type II was performed on histological sections of the explants. Analysis of the immunostained sections appears to indicate the presence of stronger staining with the collagen type II antibody when compared to collagen type I, which is in agreement with the real-time PCR analyses, although the concluding evidence of this is complicated by the background staining of the hydrogel. Collagen type I expression is linked to the dedifferentiation process that occurs in $2 \mathrm{D}$ culture and the balance between collagen types I and II is frequently used as an indicator of the cartilage type formed. The presence of some collagen type I may relate to the redifferentiation process, where collagen type I expression starts to decrease and in turn collagen type II and aggrecan are produced. The noticed stronger staining for collagen type II suggests the deposition of a hyaline-like ECM by the human articular chondrocytes encapsulated in gellan gum hydrogels after implantation in the back of nude mice.

Taken together, the results from this work have shown that gellan gum hydrogels are adequate supports for the growth and differentiation of human articular chondrocytes when implanted subcutaneously in nude mice, giving rise to the formation of a hyaline like extracellular matrix. However, some aspects should be considered when optimizing this system for further in vivo applications. It is likely that the new cartilage tissue formed would benefit from higher cell concentrations and higher implantation periods in order to achieve the formation of a tissue with improved potential. The mechanical properties of these systems should also be more thoroughly considered when approaching a loadbearing in vivo scenario. However, the synthesis profiles of collagen type II and aggrecan indicate that the mechanical support that is lacking at an initial stage may in part be assured by the newly formed ECM. Finally, it can be referred that tests with alternative cell sources such as stem cells should also be pursued. Given the scarcity of cartilage samples with potential use in patients and the immunological adversities that may arise from the use of allogenous material, such an improvement might increase the potential of gellan gum hydrogels as compared to other systems proposed for these applications.

\section{Conclusions}

In this work, gellan gum hydrogels were used to encapsulate and support human chondrocyte development upon in vivo subcutaneous implantation in nude mice. The results were quite promising in terms of the generation of a functional cartilage tissue-engineered construct. The human chondrocytes proliferated during the 4 weeks of the experiments and deposited a hyaline-like extracellular matrix, typical of native articular cartilage. Collagen type II and aggrecan showed increasing profiles, the results being coherent in the analyses performed. The maintenance of the hyaline cartilage phenotype was suggested by the absence or decrease of collagen type I and collagen type $\mathrm{X}$. The in vivo performance of these systems so far, along with previous data, suggests their further study in larger animals and the testing of different parameters towards the development of a fully functional cartilage tissue-engineered construct to be applied clinically.

\section{Acknowledgements}

J. T. Oliveira would like to acknowledge the Portuguese Foundation for Science and Technology (FCT) for his grant (SFRH/BD17135/2004). The authors would like to thank the patients at Hospital de S. Marcos, Braga, Portugal, for the donation of the biological samples and the medical staff for their help and support. The authors would also like to thank the Institute for Health and Life Sciences (ICVS), University of Minho, Braga, Portugal, for allowing the use of their research facilities. This work was carried out under the scope of European NoE EXPERTISSUES (Project No. NMP3-CT-2004-500283) and partially supported by the European Project HIPPOCRATES (No. STRP 505758-1).

\section{References}

Andersson H, van den Berg A. 2004; Microfabrication and microfluidics for tissue engineering: state of the art and future opportunities. Lab on a Chip 4(2): 98-103. 
Benya PD, Shaffer JD. 1982; Dedifferentiated chondrocytes reexpress the differentiated collagen phenotype when cultured in agarose gels. Cell 30(1): 215-224.

Cancedda R, Descalzi Cancedda F, Castagnola P. 1995; Chondrocyte differentiation. Int Rev Cytol 159: 265-358.

Cao Y, Vacanti JP, Paige KT, et al. 1997; Transplantation of chondrocytes utilizing a polymer-cell construct to produce tissueengineered cartilage in the shape of a human ear. Plast Reconstr Surg 100(2): 297-302.

Capito RM, Azevedo HS, Velichko YS, et al. 2008; Self-assembly of large and small molecules into hierarchically ordered sacs and membranes. Science 319(5871): 1812-1816.

Cortivo R, Brun P, Rastrelli A, et al. 1991; In vitro studies on biocompatibility of hyaluronic acid esters. Biomaterials 12(8): 727-730.

Crawford A, Dickinson SC. 2004; Methods in Molecular Biology: Biopolymer Methods in Tissue Engineering. Humana Press: Totowa, NJ, 2004.

Freed LE，Vunjak-Novakovic G，Langer R. 1993; Cultivation of cell-polymer cartilage implants in bioreactors. J Cell Biochem 51(3): 257-264.

Ge Z, Hu Y, Heng BC, et al. 2006; Osteoarthritis and therapy. Arthrit Rheum 55(3): 493-500.

Girkontaite I, Frischholz S, Lammi P, et al. 1996; Immunolocalization of type $\mathrm{X}$ collagen in normal fetal and adult osteoarthritic cartilage with monoclonal antibodies. Matrix Biol 15(4): 231-238.

Gomes ME, Azevedo HS, Moreira AR, et al. 2008; Starch-poly( $\varepsilon$ caprolactone) and starch-poly(lactic acid) fibre-mesh scaffolds for bone tissue engineering applications: structure, mechanical properties and degradation behaviour. J Tissue Eng Regen Med 2(5): 243-252.

Grasdalen H, Smidsrod O. 1987; Gelation of gellan gum. Carbohydr Polymers 7(5): 371-393.

Hangody L, Fules P. 2003; Autologous osteochondral mosaicplasty for the treatment of full-thickness defects of weight-bearing joints: ten years of experimental and clinical experience. $J$ Bone Joint Surg Am 85(suppl 2): 25-32.

Hendriks J, Riesle J, van Blitterswijk CA. 2007; Co-culture in cartilage tissue engineering. J Tissue Eng Regen Med 1(3): $170-178$.

http://frodo.wi.mit.edu/.

Hubbell JA, Massia SP, Desai NP, et al. 1991; Endothelial cellselective materials for tissue engineering in the vascular graft via a new receptor. Nat Biotech 9(6): 568-572.

Hunt SA, Jazrawi LM, Sherman OH. 2002; Arthroscopic management of osteoarthritis of the knee. J Am Acad Orthop Surg 10(5): 356-363.

Jackson RW, Dieterichs C. 2003; The results of arthroscopic lavage and debridement of osteoarthritic knees based on the severity of degeneration. Arthrosc J Arthrosc Rel Surg 19(1): 13-20.

Jakob RP, Franz T, Gautier E, et al. 2002; Autologous osteochondral grafting in the knee: indication, results, and reflections. Clin Orthop Relat Res 401: 170-184.

Kafienah W, Sims TJ. 2004; Methods in Molecular Biology: Biopolymer Methods in Tissue Engineering. Humana Press: US, Totowa, NJ, 2004.

Kang KS, Veeder GT, Mirrasoul PJ, et al. 1982; Agar-like polysaccharide produced by a Pseudomonas species - production and basic properties. Appl Environ Microbiol 43(5): 1086-1091.

Knudson CB, Knudson W. 2001; Cartilage proteoglycans. Semin Cell Dev Biol 12(2): 69-78.

Langer R, Vacanti JP. 1993; Tissue engineering. Science 260(5110): 920-926.
Malafaya PB, Santos TC, van Griensven M, et al. 2008; Morphology, mechanical characterization and in vivo neo-vascularization of chitosan particle aggregated scaffolds architectures. Biomaterials 29(29): 3914-3926.

Mano JF, Reis LR. 2007; Osteochondral defects: present situation and tissue engineering approaches. J Tissue Eng Regen Med 1(4): 261-273.

Mao R, Tang J, Swanson BG. 2000; Texture properties of high and low acyl mixed gellan gels. Carbohydrate Polym 41(4): 331-338.

Mooney DJ, Mazzoni CL, Breuer C, et al. 1996; Stabilized polyglycolic acid fibre-based tubes for tissue engineering. Biomaterials 17(2): 115-124.

Moroni L, de Wijn JR, van Blitterswijk CA. 2006; 3D fiber-deposited scaffolds for tissue engineering: influence of pores geometry and architecture on dynamic mechanical properties. Biomaterials 27(7): 974-985.

Oliveira JM, Rodrigues MT, Silva SS, et al. 2006; Novel hydroxyapatite/chitosan bilayered scaffold for osteochondral tissue-engineering applications: scaffold design and its performance when seeded with goat bone marrow stromal cells. Biomaterials 27(36): 6123-6137.

Oliveira JT, Correlo VM, Sol PC, et al. 2008a; Assessment of the suitability of chitosan/polybutylene succinate scaffolds seeded with mouse mesenchymal progenitor cells for a cartilage tissue engineering approach. Tissue Eng A 14(10): 1651-1661.

Oliveira JT, Santos TC, Martins L, et al. 2008d; Gellan gum injectable hydrogels for cartilage tissue engineering applications: in vitro studies and preliminary. In Vivo Eval Submitted.

Oliveira JT, Sousa RA, Reis RL. 2008b; Gellan gum based hydrogels for use in regenerative medicine and tissue engineering, its system, and processing devices (patent pending).

Oliveira JT, Martins L, Picciochi R, et al. 2009c; Gellan gum: a new biomaterial for cartilage tissue engineering applications. Journal of Biomedical Materials Research-Part A (J Biomed Mater Res A) (Accepted for Publication).

Rowley JA, Madlambayan G, Mooney DJ. 1999; Alginate hydrogels as synthetic extracellular matrix materials. Biomaterials 20(1): 45.

Sittinger M, Bujia J, Minuth WW, et al. 1994; Engineering of cartilage tissue using bioresorbable polymer carriers in perfusion culture. Biomaterials 15(6): 451-456.

Temenoff JS, Mikos AG. 2000; Review: tissue engineering for regeneration of articular cartilage. Biomaterials 21(5): 431-440.

Kirsch T, Swoboda B, von der Mark K. 1992; Ascorbate independent differentiation of human chondrocytes in vitro: simultaneous expression of types I and X collagen and matrix mineralization. Differentiation 52(1): 89-100.

Tuzlakoglu K, Bolgen N, Salgado AJ, et al. 2005; Nano- and microfiber combined scaffolds: a new architecture for bone tissue engineering. J Mater Sci Mater Med 16(12): 1099-1104.

Vacanti CA, Vacanti JP. 1994; Bone and cartilage reconstruction with tissue engineering approaches. Otolaryngol Clin N Am 27(1): 263-276.

Videki B, Klebert S, Pukanszky B. 2005; Grafting of caprolacton to cellulose acetate by reactive processing. Eur Polym J 41(8): 1699-1707.

von der Mark K, Kirsch T, Nerlich A, et al. 1992; Type X collagen synthesis in human osteoarthritic cartilage. Indication of chondrocyte hypertrophy. Arthrit Rheum 35(7): 806-811.

Vunjak-Novakovic G, Freed LE. 1998; Culture of organized cell communities. Adv Drug Deliv Rev 33(1-2): 15-30. 